Myriam Britto dos Santos

\title{
Ergonomia, Carga mental de Trabalho, Riscos e Prevenção
} de Acidentes: 0 Caso do Trabalhador em Histotécnica

Tese apresentada ao Programa de Pósgraduação em Design da PUC-Rio como requisito parcial para obtenção do título de Doutor em Design.

Orientadora: Anamaria de Moraes Co-orientadora: Valéria Barbosa Gomes

Rio de Janeiro novembro de 2007 
Myriam Britto dos Santos

\title{
Ergonomia, Carga mental de Trabalho, Riscos e Prevenção de Acidentes: 0 Caso do Trabalhador em Histotécnica
}

\author{
Tese apresentada como requisito \\ parcial para obtenção do grau de \\ Doutor pelo Programa de Pós- \\ Graduação em Design do \\ Departamento de Artes \& Design do \\ Centro de Teologia e Ciências \\ Humanas. Aprovada pela Comissão \\ Examinadora abaixo assinada.
}

Profa. Dra. Anamaria de Moraes Presidente/PUC-Rio

Profa. Dra. Vera Lucia M. dos S. Nojima PUC-Rio

Prof. Dr. Heliomar de Azevedo Valle UNI-Rio

Prof. Dr. Carlos Alberto Basílio de Oliveira UNI-Rio

Prof. Dr. Nilton Pinto Ribeiro Filho UFRJ

Prof. Dr. Paulo Fernando Carneiro de Andrade Coordenador Setorial do Centro de Teologia e Ciências Humanas

PUC-Rio

Rio de Janeiro, 29 de novembro de 2007 
Todos os direitos reservados. É proibida a reprodução total ou parcial do trabalho sem autorização da universidade, da autora e do orientador.

\section{Myriam Britto dos Santos}

Psicóloga, Bacharel e Licenciada em Psicologia graduada pela Universidade Gama Filho, 1979. Especialista em Ergonomia, Centro de Pósgraduação em Psicologia Aplicada do ISOP/FGV, 1982. Mestre em Psicologia, Área de Concentração Processos Cognitivos, Instituto de Psicologia da UFRJ, 2001. Ergonomista Certificada pela Câmara Técnica de Certificação do Ergonomista Brasileiro - da Associação Brasileira de Ergonomia. Sócia Fundadora da ABERGO. Pesquisadora bolsista do $\mathrm{CNPq}$ de apoio técnico (1 A CNPq - 01/98 07/99), mestrado (IP/UFRJ, de 1998 - 2001), doutorado (Processo 1408382004-0) com foco em temas relacionados à Psicologia Cognitiva, Ergonomia Psicológica, à carga de trabalho, prevenção de acidentes, sofrimento psíquico e humanização do trabalho.

Ficha Catalográfica

\section{Santos, Myriam Britto dos}

Ergonomia, carga mental de trabalho, riscos e prevenção de acidentes: o caso do trabalhador em histotécnica / Myriam Britto dos Santos; orientador: Anamaria de Moraes/coorientador Valéria Barbosa Gomes - 2007 232 f.: il. ; $30 \mathrm{~cm}$

Tese (Doutorado em Design)-Pontifícia Universidade Católica do Rio de Janeiro, Rio de Janeiro, 2007.

Inclui bibliografia

1. Artes - Teses. 2. Ergonomia. 3. Carga mental de trabalho. 4. Método L.E.S.T. 5. Carga de trabalho. 6. Sofrimento psíquico. 7. Micrótomo rotativo. 8. Histotécnica. I. Moraes, Anamaria. II. Pontifícia Universidade Católica do Rio de Janeiro. Departamento de Artes. III. Título. 
A minha mãe, Lucia, que me faz sentir que o amor transborda como missão no mundo, que o amor vive eterno à parte de um mundo de ilusões. 


\section{Agradecimentos}

Á minha orientadora, Profa. Dra.Anamaria de Moraes, primeira Fellow da IEA, em toda a América Latina, meu reconhecimento e gratidão pela presença afetiva e instigante orientação imantada pelo conhecimento e pela valorização da pessoa humana no trabalho.

Á Profa. Dra. Valéria Barbosa Gomes, Professora Doutora em Saúde Coletiva pela Universidade do Estado do Rio de Janeiro, minha co-orientadora, pelo incentivo e contribuição enriquecedora no desenvolvimento desta pesquisa.

Ao Ilustre Prof. Dr. Carlos Alberto Basilio de Oliveira, Professor Titular da Disciplina de Anatomia Patológica do Hospital Universitário Gaffrée e Guinle, pelo acolhimento, gentileza e, apoio incondicional em todas as fases desta pesquisa.

Ao Prof. Dr. Heliomar de Azevedo Valle, um ser de luz, sintonizado com a inteligência e sensibilidade, minha eterna amizade.

Aos Histotécnicos do Laboratório de Anatomia Patológica do Hospital Universitário Gaffrée e Guinle, pela confiança e colaboração, minha profunda admiração como pessoas e trabalhadores pelo nobre trabalho que realizam.

À direção do Hospital Universitário Gaffrée e Guinle, onde a pesquisa foi realizada permitindo a coleta de dados imprescindíveis.

Ao Prof. Dr. Ir. Jan Dul, Professor of Ergonomics Management da RSM Erasmus University, pelas sugestões de leituras e consultas a bases de dados relevantes que me foram dadas por ocasião do $5^{\circ}$ Ergodesign, $5^{\circ}$ Congresso Internacional deErgonomia e Usabilidadede Interfaces Humano-Tecnologia: Produtos, Informação, Ambiente Construído, Transporte, LEUI-PUC-Rio em 2005.

Ao Prof. Dr. Nilton Pinto Ribeiro Filho, uma pessoa rara, que reúne sabedoria e ações determinadas numa trajetória dedicada à investigação científica, meu carinho e admiração.

Aos Professores do Curso de Doutorado em Design, pelo acesso às leituras e pelos relevantes enfoques teóricos apresentados em seus cursos.

Aos funcionários da Secretaria do Departamento de Artes e Design, (em ordem alfabética), Aylton Romão Pessanha, Chrystiane Oliveira Alves, Claudio Roberto Souza Barros, Josué Alves Assis Silva, Marlon Santos de Oliveira, Monica Pinho do Nascimento, Rodrigo Oliveira Medeiros, Romário César de Sousa, Verônica da E. Silva Paiva, pela solicitude que dispensaram a minha pessoa. 
A todos os amigos do Curso de Doutorado e do Laboratório de Ergonomia e Usabilidade de Interfaces em Sistemas Humano-Tecnologia- LEUI-PUC-Rio pelos momentos partilhados.

Ao Dr. Rodrigo Panno Basílio de Oliveira, Professor Assistente de Anatomia Patológica da UNI-RIO e da PUC-Rio e aos residentes do Laboratório de Anatomia Patológica, (em ordem alfabética), Dr. André Luiz da Rocha Azevedo, Dra. Maria Clara Reder de Sousa, Dra. Roberta Acar Pereira, e Dra. Verônica Goulart Moreira, pelo carinho e colaboração durante as visitas realizadas ao Hospital Universitário Gaffrée e Guinle.

Aos funcionários do Laboratório de Anatomia Patológica do Hospital Universitário Gaffrée e Guinle, em especial, ao secretário Ronildo Bezerra dos Santos.

À Profa. Dra. Vera Lúcia Moreira dos Santos Nojima pela presença simpática e incentivadora em todos os momentos.

Ao Prof. Dr. Carlos Américo Alves Pereira pelas sugestões esclarecedoras e afável disponibilidade para troca de idéias.

Ao CNPq pelo apoio financeiro na forma de bolsa de estudo. 
A Deus e aos mentores espirituais.

À minha família e em especial aos meus pais, Derly Antonio dos Santos (in memoriam), minha incomensurável saudade e Lucia Britto dos Santos, minha mãe, terna e eterna em meu coração.

À minha filha Letícia, um sonho de Deus, que resplandece de alegria em minha vida. 


\section{Resumo}

Santos, Myriam Britto dos; Moraes, Anamaria de. (orientadora), Gomes, Valéria B. (co-orientadora) Ergonomia, Carga Mental de Trabalho, Riscos e Prevenção de Acidentes: O Caso do Trabalhador em Histotécnica. Rio de Janeiro, 2007. Pp.232. Tese de Doutorado - Departamento de Artes \& Design, Pontifícia Universidade Católica do Rio de Janeiro.

Em uma pesquisa descritiva e de estudo de campo, buscou-se avaliar quais indicadores de carga mental $(\mathrm{CM})$ de trabalho são predominantes para a produção de acidentes em uma tarefa de microtomia. A carga mental depende das exigências da tarefa e do grau de mobilização do sujeito, da fração de sua capacidade de trabalho que ele investiu na tarefa, considerando-se que o grau de prazer e satisfação no trabalho pode variar em função da natureza da tarefa executada. Partiu-se da hipótese que os fatores de carga mental de trabalho associados ao risco de acidentes a que está exposto o operador no manuseio do micrótomo rotativo representam fonte de sofrimento psíquico. Os sinais de sofrimento psíquico podem ser vinculados à realização de tarefas consideradas perigosas e, muitas atividades de trabalho podem ocasionar desgastes e custos para o indivíduo de ordem física, mental, emocional e afetiva. Foi relevante examinar quais foram os fatores organizacionais referentes às condições ambientais e aos horários de trabalho, para uma ação preventiva, em conjunto aos fatores predominantes de carga mental. O suporte metodológico da pesquisa envolveu a elaboração de um questionário adaptado do método L.E.S.T. (Laboratório de Economia e Sociologia do Trabalho) que avalia a carga mental a partir de quatro indicadores, a saber, constrangimento de tempo; complexidade/rapidez; atenção, e minúcia. Os resultados obtidos mostraram que a variável complexidade-rapidez, minúcia e atenção podem ser consideradas como efetores para carga mental. A variável denominada fatores psicossociais não mostrou eficácia durante este processo de análise. Um aspecto relatado está associado aos acidentes produzidos durante a operação do equipamento e sua manutenção.

Palavras-chave:Ergonomia, Carga mental de trabalho, Método L.E.S.T., Carga de trabalho, Sofrimento psíquico, Micrótomo rotativo, Histotécnica. 


\section{Abstract}

Santos, Myriam Britto dos; Moraes, Anamaria de (Advisor), Gomes, Valéria B. (Co-Advisor) Ergonomics, Mental Workload, Risks, and Accident Prevention: the case of histotechnicians. Rio de Janeiro, 2007. Pp.232. Doctorate Thesis - Departamento de Artes \& Design, Pontifícia Universidade Católica do Rio de Janeiro.

In a descriptive research and in a field study, we evaluated which indicators of mental workload are dominant factors in accident production in a microtomy task. The mental workload depends on the task demands and on the degree of mobilization of the worker, which is the fraction of his/her work capacity invested in the task considering itself that the degree of pleasure and satisfaction in work could vary as a function of the nature of executed task.From the hypothesis that the factors of mental workload associated to accident risks to which operators are exposed during handling of a rotative microtome represent a source of psychic suffering. The signals of psychic suffering could be related to the accomplishment of tasks considered as dangerous and many work activities could cause physical, mental, emotional and affective wearing and costs to individuals. Examining which organizational factors are related to environmental conditions and to working hours was relevant to a preventive action, as well as the dominant factors of mental workload. The research methodology involved the preparation of a questionnaire adapting the L.E.S.T. (Laboratoire d'Economie et Sociologie du Travail) method that evaluates the mental workload by four indicators, which are time constraint; complexity/ swiftness; attention; and detail. The results showed that complexity/swiftness, detail and attention indicators could be considered as effective indicators of mental workload. The indicator psychosocials factors did not presented efficacy in this analytical procedure. A reported feature is associated to accidents happening during the handling of equipment and its maintenance.

\section{Key words}

Ergonomics, Mental workload, L.E.S.T. method, Workload, psychic suffering, rotative microtome, histotechnology. 


\section{Sumário}

$\begin{array}{ll}1 \text { - Introdução } & 18\end{array}$

2- Custos humanos e cargas de trabalho: carga física, cognitiva e psíquica

2.1- A carga mental de trabalho e seus métodos de medida 37

2.2- Procedimentos subjetivos 53

2.2.1-Procedimentos subjetivos unidimensionais 53

2.2.2- Procedimentos subjetivos multidimensionais 56

2.2.3. Medidas fisiológicas 63

2.3- Carga mental de trabalho e fadiga 64

2.3.1-A norma ISO 10075

2.4- Sofrimento psíquico e humanização do trabalho 72

2.5- A carga psíquica de trabalho $\quad 75$

2.6- Conclusão do capítulo $\quad 77$

3- Concepções sobre o paradigma do erro humano, a gestão de riscos e a gestão de acidentes.

3.1- A noção de gestão de riscos $\quad 87$

3.2- Conceituação de acidente, incidente e quase acidente 88

3.3- Os modelos de estudo de acidentes $\quad 92$

3.3.1- Um resumo dos modelos de acidentes a partir de Melia e 93

$\begin{array}{ll}\text { colaboradores (1998) } & 93 \\ \text { 3.3.1.1- Modelos explicativos de acidentes de inspiração mecanicista } & 93\end{array}$

3.3.1.1 a- Os modelos de dominó e de liberação de energia 93

3.3.1..1b- Os modelos de interação humano -máquina 96

3.3.1.1c- Os modelos de erro humano $\quad 97$

3.3.1.2- Os modelos explicativos de inspiração psicossociológica 101

3.3.1.2 a- O modelo comportamental 102

3.3.1.2b- O modelo cognitivo 104

3.3.1.2c- O modelo de perspectiva da personalidade 107

$\begin{array}{ll}3.3 .1 .2 \mathrm{~d} \text { - O modelo de perspectiva sociológica } & 107\end{array}$

3.4- Conclusão do capítulo 113

4- O micrótomo rotativo $\quad 115$

4.1- Conclusão do capítulo 123

5 - Métodos e Técnicas da Pesquisa 124 
5.1-Delimitação da pesquisa $\quad 124$

5.2-Métodos e técnicas 126

5.3-Procedimentos 128

6- Apresentação e análise dos resultados 131

6.1- Fluxograma das atividades da tarefa do histotécnico: Etapas de 137 processamento do material a ser examinado

162

6.2-Análise qualitativa

\begin{tabular}{ll} 
6.2.1. Satisfação no trabalho & 175 \\
\hline
\end{tabular}

6.2.2. Relato dos acidentes 176

6.3. Conclusão do capítulo 179

$\begin{array}{ll}\text { 7- Discussão } & 181\end{array}$

8-Conclusão Final 190

8.1-Conclusões 190

8.2-Recomendações 194

8.3-Desdobramentos da pesquisa 195

9- Referências bibliográficas 196

Anexos

Anexo I: Cronograma de desenvolvimento da pesquisa

Anexo II-Trabalhos publicados

Anexo III: Questionário de avaliação do trabalho do histotécnico

Anexo IV: Autorização para uso da imagem

Anexo V: Termo de compromisso livre e esclarecido (modelo)

Anexo VI: Produtos químicos

Anexo VII: Resolução do comitê de Ética em pesquisa

Anexo VIII: Quadro com o perfil dos participantes

Anexo IX: Normas regulamentadoras: NR 7, NR 9, NR15 (Anexo no 11, Anexo 14), NR 17 (Anexo II), NR 32, NORMA ISO 10075 


\section{Lista de Tabelas}

Tabela 1: Respostas binomiais ( $\operatorname{sim} \times$ não) para o fator 131 constrangimento de tempo.

Tabela 2: Respostas binomiais para o fator complexidade-rapidez. 132

Tabela 3: Respostas binomiais para o fator atenção. 133

Tabela 4: Respostas binomiais para o fator minúcia. 134

Tabela 5: Respostas binomiais para os fatores psicossociais. 134 


\section{Lista de Quadros}

Quadro 1: Títulos e subtítulos. 23

Quadro 2: Condições, constrangimentos, conseqüências,
resultados e qualidade do trabalho.

resultados e qualidade do trabalho.

Quadro 3: Fatores da carga de trabalho. 32

Quadro 4: Principais fatores de carga mental no trabalho 42

hospitalar.

Quadro 6: Definição dos níveis de cada uma das três definições $\quad 57$

do SWAT.

Quadro 7: Definição das seis dimensões do NASA-TLX. 59

Quadro 8: Modelo de matriz (workload profile). 62

Quadro 9: Resumo de definições do conceito de carga mental de trabalho.

Quadro 10: A pirâmide de Maslow

Quadro 11: Os modelos de dominó e elaboração de energia.

Quadro 12: Os modelos de interação humano-máquina.

Quadro 13: Os modelos de erro humano.

68

Quadro 14: O modelo comportamental.

Quadro 15: O modelo cognitivo. 109

Quadro 16: O modelo de perspectiva de personalidade. 111

Quadro 17: O modelo de perspectiva sociológica. 112

Quadro 18: Três abordagens para a gestão de acidentes. 112

Quadro 19: Distinções entre carga mental e fadiga e subcarga e 181 sobrecarga.

Quadro 20: Exemplos de processos cognitivos.

184

Quadro 21: Níveis de processamento de informação. 185

Quadro 22: Aspectos relacionados com a atenção no trabalho. 186 


\section{Lista de Figuras}

Figura 1: Micrótomo rotativo (American Optical 820) 20

Figura 2: Representação esquemática do aumento de freqüência 31 cardíaca.

Figura 3: Determinantes de carga de trabalho. 35

Figura 4: Etapas do processo cognitivo. $\quad 40$

Figura 5: Fatores determinantes e conseqüências do trabalho 41

mental.

Figura 6: Relação hipotética entre carga mental e rendimento. 49

Figura 7: Representação de uma PRF típica. 51

Figura 8: Representação de uma POC típica. $\quad 51$

Figura 9: Micrótomo rotativo de 1905.

Figura 10: Detalhamento do Micrótomo American Optical 820.

Figura 11: Micrótomo Shandon 315. 119

Figura 12: Micrótomo Shandon Finesse 325.

Figura 13: Detalhamento do Shandon Finesse 325.

Figura 14: Micrótomo Shandon Finesse (R). 120

Figura 15: Micrótomo rotativo MRPO3-LUPE. 121

Figura 16: Autocut 2055, Histocut 820, entre outros. 122

Figura 17: Respostas em porcentagem das variáveis de carga 136 mental de trabalho.

Figura 18: Aparelho processador automático de tecido. 138

Figura 19: Processo de inclusão do material. 139

Figura 20: Histotécnico retirando do interior dos cassetes de 140

plástico os fragmentos de tecidos.

Figura 21: Material parafinado. 140

Figura 22: Fragmentos de tecidos. $\quad 140$

Figura 23: Material parafinado colocado no fundo do molde de 141

metal.

Figura 24: Conjunto molde de mental, cassete de plástico e 141

material parafinado.

Figura 25: Bloco preparado. 141

Figura 26: Bloco de parafina colocado no micrótomo. 142

Figura 27: Detalhe do ajuste do bloco. 142

Figura 28: Outro ângulo do ajuste do bloco 143

Figura 29: Processo de microtomia. 143

Figura 30: Processo de microtomia/giro da manivela. 143

Figura 31: Retirada da fita de parafina. 144

Figura 32: Fita parafinada é segura pelos dedos do histotécnico. 144

Figura 33: Técnico segura uma lâmina de vidro. 145

Figura 34: Identificação da lâmina. $\quad 145$

Figura 35: Lâminas identificadas. $\quad 146$

Figura 36: Histotécnico regulando o bloco no micrótomo. 147

Figura 37: Movimento sincronizado com as mãos. 147

Figura 38: Histotécnico manipulando o micrótomo. 147 
Continuação.

Figura 39: Extensão do braço direito. 148

Figura 40: Histotécnico levando a fita de parafina do micrótomo 148

para o banho-maria.

Figura 41: Histotécnico manipula fita de parafina. 148

Figura 42: Histotécnico estica com uma pinça a fita de parafina. 149

Figura 43: Histotécnico esticando a fita de parafina na água do 149

banho-maria com as duas mãos.

Figura 44: Histotécnico pega uma lâmina de vidro. 150

Figura 45: Histotécnico trabalhando lado a lado. $\quad 150$

Figura 46: Inclusão/microtomia (detalhe do anterior). 151

Figura 47: Cooperação entre os postos de inclusão e microtomia. 151

Figura 48: Histotécnico colocando o material no molde. 152

Figura 49: Histotécnico em primeiro plano realizando a inclusão 152

de material.

Figura 50: Comunicação e desvio do olhar da atividade. 153

Figura 51: Histotécnico prossegue a atividade. 153

Figura 52: Histotécnico afiando a navalha. 154

Figura 53: Histotécnico escolhendo reagentes e corantes. 154

Figura 54: Processo de coloração de rotina. $\quad 155$

Figura 55: Histotécnico trabalhando na sala de coloração. 156

Figura 56: Histotécnico retira a lâmina da cuba com xilol. 156

Figura 57: Histotécnico segura a lâmina com material corado. 156

Figura 58: Histotécnico coloca uma gota de bálsamo sobre a 157

lâmina.

Figura 59: Histotécnico coloca a lamínula. 157

Figura 60: Histotécnico montando a lâmina de vidro. 158

Figura 61: Histotécnico arrumando as lâminas. 158

Figura 62: Lâminas montadas. 159

Figura 63: Histotécnico faz uma apreciação do resultado. 159

Figura 64: Médicos examinando as lâminas. $\quad 159$

Figura 65: Técnico de laboratório separando as lâminas. $\quad 160$

Figura 66: Detalhe do anterior. $\quad 160$

Figura 67: Seqüência para realização de algumas fases da tarefa 161

de microtomia (micrótomo Shandon Finesse 325).

Figura 68: Outra seqüência da tarefa de microtomia (micrótomo 162

Shandon Finesse 325).

Figura 69: Ferimento produzido nos dedos pela manipulação de um micrótomo. 
"Sempre há uma razão para se viver.

Podemos nos elevar sobre nossa ignorância, podemos nos descobrir como criaturas de perfeição, inteligência e habilidade. Podemos ser livres! Podemos aprender a voar!"

(Richard Bach, de Fernão Capelo Gaivota, Liberdade)

“A Ergonomia é uma tomada de posição em prol do ser humano e sua dignificação; não há coerência hoje em dia no fato de se conseguirem engenhos capazes de transcender nosso planeta e não se conseguirem produzir máquinas e ferramentas capazes de proteger seus operadores contra riscos de vida, de mutilações, de doenças profissionais ou mesmo contra esforços desgastantes ou condutas neurotizantes". Franco Lo Presti Seminério (1976), In memoriam 